\title{
Opportunities in space life sciences
}

\author{
Jason Dowling ${ }^{1,2,3,4,5} \cdot$ Anatoly Rosenfeld $^{3} \cdot$ James Waldie $^{6,7} \cdot$ Ilana Feain $^{8}$
}

Published online: 10 June 2019

(c) Australasian College of Physical Scientists and Engineers in Medicine 2019

There are very positive signs that in the next few decades space travel for scientific understanding, exploration, in-situ resource utlilisation, tourism, and eventual settlement (lunar and Martian habitation) will become reality.

Some of the most exciting developments are being led by a resurgence in the US space budget. The recently announced United States National Aeronautics and Space Administration (NASA) Artemis lunar exploration program is aiming to return the first female and next male astronaut to the Moon's south pole by 2024 and follow this with the establishment of sustainable missions by 2028 [1]. Once lunar habitation is established NASA plans to send astronauts to Mars [2]. To support these missions NASA is leading the construction of a modular Lunar Orbital Platform-Gateway space station which would support human and robotic exploration of the moon's surface and other missions. The European, Chinese and Russian space agencies have also outlined plans for lunar settlement. There is a high level of collaboration with commercial and international partners and many of these programs.

Jason Dowling

Jason.Dowling@csiro.au

1 CSIRO Australian E-Health Research Centre, Herston, QLD, Australia

2 Faculty of Medicine, University of New South Wales, Sydney, NSW, Australia

3 Centre for Medical Radiation Physics, University of Wollongong, Wollongong, NSW, Australia

4 School of Mathematical and Physical Sciences, University of Newcastle, Newcastle, NSW, Australia

5 Institute of Medical Physics, University of Sydney, Sydney, NSW, Australia

6 Human Aerospace Pty Ltd, Adelaide, SA, Australia

7 School of Engineering, Royal Melbourne Institute of Technology, Melbourne, VIC, Australia

8 CSIRO Astronomy \& Astrophysics, Marsfield, NSW, Australia
The space sector continues to grow strongly-the number of international space agencies currently sits around 75, with fourteen of these having sovereign launch capabilities. Agencies from the US (NASA), Russia (Roscosmos), China (CNSA), European (ESA), Japan (JAXA), India (ISRO) have already conducted orbital missions to the Moon. New Zealand (2016) and Australia (2018) have recently created space agencies.

Another recent shift in the space landscape has been the emergence of private companies with more commercially focused, cost effective and agile approaches, including SpaceX, Blue Origin, and Virgin Galactic. Israel recently became the first country to send a privately funded craft (the Beresheet) into lunar orbit. Blue Origin is planning to return humans to the Moon by 2024. Over 650 people have signed up for Virgin Galactic flights [3] and Space-X has reportedly sold seats on its BFR rocket to the first private customers to travel around the moon in 2023 [4].

Robotics will play an important role in this new era in space exploration with human travel and colonization being the ultimate goal. But space is a dangerous environment for humans, particularly for long duration travel. The most serious challenge involves exposure to high levels of ionising radiation (increasing risks of cancer, cataracts, cardiac damage, etc). In addition, based on studies of astronauts living in the microgravity conditions of the ISS, the space environment can lead to many of the same health issues that occur in an exponentially ageing human, including bone loss, muscle atrophy, kidney stones, eye damage from increased intracranial pressure, altered immunology, and sleeping difficulties. Astronauts are at risk of decompression sickness and suffer from decreased motor co-ordination after long duration travel [5]. Space travel involves living in a hostile closed environment with the risk of environmental hazards and changes to the behaviour of micro-organisms. Space travellers may also be isolated and confined within a small area which increases the risk of psychosocial issues (such as depression, conflict, anxiety, fatigue and team cohesion). Growing food in a microgravity environment for nutritional requirements is also difficult and medications can become 
ineffective or toxic due to long term storage. An additional problem with exploration, mining and settlement is the exposure to lunar dust (which may cause silicosis and equipment failure), and eventually Martian dust (which is both a mechanical irritant and a chemical poison) [6].

The hazards of space travel also extend to equipment and computer hardware which needs to cope with huge temperature changes (on the Moon this can range from -173 to $127^{\circ} \mathrm{C}$ depending on the amount of sunlight [7]; a lack of airflow to allow heat to dissipate; and the need to harden equipment against radiation and microgravity changes (for example, tin can form whiskers in a vacuum leading to equipment failure). Batteries for the International Space Station currently require extensive testing and flight certification before use. Despite this, some exciting recent developments have occurred with in-orbit manufacturing, 3D printing and robotics.

Australia has a long history of involvement in space and was only the third nation to launch a satellite (WRESAT) from its own territory. Australia has collaborated with NASA for over 50 years including providing support for the Apollo 11 moon landing. Apart from the new Australian Space Agency there are several additional federal and state government investments in Australia relating to space technology including the CSIRO Space Technology Future Science Platform and the SmartSat Cooperative Research Centre aiming to leverage Australian expertise in space sciences.

Australia and New Zealand have strong existing expertise in the development of medical technologies (such as ultrasound imaging) and remote and polar medicine [8] which provide us with a competitive advantage. Some examples of expertise within Australian universities include microdosimetry for space applications [9] (UoW), human factors (UCQ), stem cells in space (UNSW), space medicine (UTas) and space suit design (RMIT). This expertise extends to autonomous and artificially intelligent devices and there are likely opportunities in medical technologies for human health during long duration trips due to a lack of equipment, expertise, environmental differences, and communication latency issues (the two-way time delay is as high as $44 \mathrm{~min}$ for Earth to Mars).

Research and technology for space travel has traditionally translated to benefits for life on Earth. Space health related inventions with commercial success include memory foam, air purifiers and even the Bowflex gym. New techniques for existing equipment have been developed in space (such as new ultrasound techniques for remote environments and methods for water purification), along with innovative new applications of space technology (such as the Australian developed SkinSuit [10] derived compression garments for elite athletes, heart pump design, polymer coatings for cardiac resynchronization devices and ingestible thermometers for body temperature monitoring). Future benefits might involve microgravity bone density research that could help prevent age related disease and agricultural innovation in harsh environments.

In summary the space sector is undergoing rapid growth and there are many opportunities for Australia and New Zealand to collaborate on projects with huge future impact. To take advantage of these future opportunities we need to network, collaborate, support each other, and be advocates in space life sciences across Australasian government, academia and industry.

\section{References}

1. NASA (2019) NASA Taps 11 American Companies to Advance Human Lunar Landers. https://www.nasa.gov/press-release/nasataps-11-american-companies-to-advance-human-lunar-landers/

2. NASA (2018) National Space Exploration Campaign Report. Retrieved from https://www.nasa.gov/sites/default/files/atoms/ files/nationalspaceexplorationcampaign.pdf

3. Jeff F (2017) All booked: virgin Galactic says suborbital spaceflights are full until 2021-SpaceNews.com. SpaceNews website https://spacenews.com/all-booked-virgin-galactic-says-suborbital -spaceflights-are-full-until-2021/. Accessed 31 May 2019

4. Kenneth Chang. (2018). Meet SpaceX's First Moon Voyage Customer, Yusaku Maezawa-The New York Times. New York Times website https://www.nytimes.com/2018/09/17/science/ spacex-moon-tourism-passenger.html. Accessed 31 May 2019

5. Moore ST, Dilda V, Morris TR, Yungher DA, MacDougall HG, Wood SJ (2019) Long-duration spaceflight adversely affects post-landing operator proficiency. Sci Rep 9(1):2677. https://doi. org/10.1038/s41598-019-39058-9

6. Jones J, Barratt M, Effenhauser R, Cockell CS, Lee P (2004) Medical issues for a human mission to Mars and Martian surface expeditions. J Br Interplanet Soc 57(3-4):144-160

7. Williams J-P, Paige DA, Greenhagen BT, Sefton-Nash E (2017) The global surface temperatures of the Moon as measured by the Diviner Lunar Radiometer Experiment. Icarus 283:300-325. https ://doi.org/10.1016/J.ICARUS.2016.08.012

8. Space Life Sciences Comittee of the Australasian Society of Aerospace Medicine (2017) Space Life Sciences: Australia's future space industry capability. https://consult.industry.gov.au/space -activities/review-of-australian-space-industry-capability/consu ltation/download_public_attachment?sqId=question.2017-0727.4002791506-publishablefilesubquestion\&uuId $=667442310$

9. Rosenfeld AB (2016) Novel detectors for silicon based microdosimetry, their concepts and applications. Nucl Instrum Methods Phys Res Sect A 809:156-170. https://doi.org/10.1016/J. NIMA.2015.08.059

10. Waldie JM, Newman DJ (2011) A gravity loading countermeasure skinsuit. Acta Astronaut 68(7-8):722-730. https://doi. org/10.1016/J.ACTAASTRO.2010.07.022 